Enfermagem Brasil 2018;17(3):190-8

\title{
ARTIGO ORIGINAL \\ Conhecimento de cuidadores formais de idosos para manter a pele do idoso livre de lesão por pressão
}

Paula Soares Carvalho*, Elizabeth Souza Silva de Aguiar, M.Sc.**, Karen Krystine Gonçalves de Brito, M.Sc. ${ }^{* \star *}$, Ester Missias Villaverde Antas ${ }^{\star \star * *}$, Smalyanna Sgren da Costa Andrade, M.Sc. ${ }^{* * * *}$, Mirian Alves da Silva, D.Sc. ${ }^{* * * * *}$, Maria Júlia Guimarães Oliveira Soares, D.Sc. ${ }^{* * \star * \star *}$

${ }^{*}$ Discente de Enfermagem, Universidade Federal da Paraíba, João Pessoa/PB, ${ }^{\star \star}$ Enfermeira, Doutoranda do Programa de Pós-Graduação de Enfermagem - PPGENF/UFPB, Membro do Grupo de Estudo e Pesquisas no Tratamento de Feridas - GEPEFE/UFPB, Universidade Federal da Paraíba, João Pessoa/PB, ${ }^{* * * E n f e r m e i r a, ~ D o u t o r a n d a ~ d o ~ P r o g r a m a ~ d e ~ P o ́ s-~}$ Graduação de Enfermagem - PPGENF/UFPB, Membro do Grupo de Estudo e Pesquisas no Tratamento de Feridas - GEPEFE/UFPB, Universidade Federal da Paraíba, João Pessoa/PB, ${ }^{* * * \star}$ Enfermeira, Especialista em UTI e Nefrologia, Membro do Grupo de Estudo e Pesquisas no Tratamento de Feridas, Universidade Federal da Paraíba, João Pessoa/PB, ${ }^{* * * * *}$ Enfermeira, Doutoranda do Programa de Pós-Graduação de Enfermagem - PPGENF/UFPB, Membro do Grupo de Estudo e Pesquisas no Tratamento de Feridas, Universidade Federal da Paraíba, João Pessoa/PB, ${ }^{* * * * * *}$ Enfermeira, Docente do departamento de Enfermagem clínica da Universidade Federal da Paraíba, João Pessoa/PB, ${ }^{* * * * * *}$ Enfermeira, Docente titular na Graduação em Enfermagem pela Universidade Federal da Paraíba (UFPB), Coordenadora do Programa de Pós-graduação em Enfermagem - PPGEnf/UFPB, Líder do Grupo de Estudos e Pesquisa em Tratamento de Feridas da Universidade Federal da Paraíba, João Pessoa/PB

Recebido em 7 de julho de 2017; aceito em 13 de março de 2018.

Endereço para correspondência: Paula Soares Carvalho, Rua Ana Cristina Rolim Machado, 253301, Residencial Funchal, Aeroclube, 10738389609 João Pessoa PB, E-mail: paaulasoares@live.com; Elizabeth Souza Silva de Aguiar: elisouaguiar@hotmail.com; Karen Krystine Gonçalves de Brito: karen_enf@yahoo.com.br; Ester Missias Villaverde Antas: ester_villaverde@yahoo.com.br; Smalyanna Sgren da Costa Andrade: smalyanna@hotmail.com; Mirian Alves da Silva: miads.enf@gmail.com; Maria Júlia Guimarães Oliveira Soares: mmjulieg@gmail.com

\section{Resumo}

Objetivos: Avaliar o conhecimento dos cuidadores formais de idosos institucionalizados sobre lesão por pressão e formas de prevenção dessa lesão. Métodos: Estudo de abordagem quantiqualitativa, realizado em duas instituições de longa permanência para idosos de João Pessoa/PB. A amostra foi de 14 cuidadores formais de idosos, entrevistados em junho e julho de 2016, por meio de roteiro estruturado, com base em guideline internacional. Para análise dos dados relacionados ao perfil demográfico e profissional foi utilizada a estatística descritiva, e para a análise das variáveis relacionadas ao conhecimento dos cuidadores três categorias foram empregadas para avaliar as respostas como: correta/completa, parcialmente correta e incorreta/incompleta. Resultados: A maioria dos entrevistados eram mulheres, com 24 a 54 anos de idade, solteiros (as), com ensino médio e metade dos entrevistados realizaram o curso de cuidador de idosos. Concernente à averiguação do conhecimento sobre as lesões por pressão, a maioria das respostas foram parcialmente corretas ou incompletas. Conclusão: Verificou-se como urgente a viabilização de intervenções educativas fundamentadas no cuidado à saúde do idoso, especificamente na capacitação dos cuidadores para prevenção das lesões por pressão.

Palavras-chave: cuidadores, idoso, instituição de longa permanência para idosos, prevenção e controle, úlcera por pressão. 


\section{Abstract \\ Knowledge of formal caregivers of elderly to keep the skin of the elderly free from pressure injury}

Objectives: To evaluate the knowledge of formal caregivers of institutionalized elderly people on pressure injuries and ways of preventing this injury. Methods: A quantitative-qualitative study, carried out in two long-term institutions for the elderly in João Pessoa/PB/Brazil. The sample was of 14 formal caregivers of the elderly, interviewed in June and July of 2016, through a structured script, based on an international guideline. Descriptive statistics were used to analyze the data related to the demographic and professional profile, and for the analysis of the variables related to the caregivers' knowledge, three categories were used to evaluate the answers as: correct/complete, partially correct and incorrect/incomplete. Results: Most of the interviewees were women, 24 to 54 years of age, single, with high school and half of the interviewees attended the caregiver course for the elderly. Concerning the investigation of knowledge about pressure injuries, most of the answers were partially correct or incomplete. Conclusion: It was verified as urgent the viability of educational interventions based on the health care of the elderly, specifically on the training of caregivers to prevent pressure injuries.

Key-words: caregivers, aged, home for the aged, prevention and control, pressure ulcer.

\section{Resumen}

Conocimiento de cuidadores formales de adultos mayores para mantener la piel del mayor libre de lesión por presión

Objetivos: Averiguar el conocimiento de cuidadores de ancianos institucionalizados en Instituciones de Larga Permanencia (ILP) e investigar los factores limitantes a la realización del cuidado a los ancianos, en lo que conciernen a las lesiones por presión. Métodos: Se trata de un estudio exploratorio con abordaje cuantitativo, realizado en 2 ILPs, en João Pessoa/PB/ Brasil. La muestra fue de 14 cuidadores, entrevistados en marzo y julio de 2016, por medio de un itinerario pre-estructurado, con base en informaciones y orientaciones establecidas por órganos de fomento nacionales e internacionales. Para el análisis de los datos relativos al perfil sociodemográfico y técnico-profesional se utilizó estadística descriptiva, mientras que la evaluación cualitativa consideró tres categorías: correcta / completa, parcialmente correcta e incorrecta / incompleta. Resultados: La mayoría de los entrevistados eran mujeres, con 24 a 54 años de edad, solteros (as), con enseñanza media y algún curso técnico relacionado a la salud. En cuanto a la averiguación del conocimiento sobre las lesiones por presión a la mayoría de las respuestas fueron parcialmente correctas o incompletas. Mientras que las limitaciones citadas remitieron principalmente a las relaciones interpersonales y sobrecarga de trabajo. Conclusión: La falta de conocimiento, junto con otras dificultades relatadas por los cuidadores, refleja de modo negativo en la asistencia prestada a esa población, acarreando consigo factores perjudiciales para la propia salud de los ancianos.

Palabras-clave: cuidadores, ancianos, institución de larga permanencia para ancianos, prevención y control, lesión por presión.

Introdução

Atualmente, o envelhecimento populacional representa um dos desafios da saúde pública de nosso país, de um lado devido à crescente demanda por serviços de saúde e por outro se depara com recursos humanos e materiais insuficientes para a adequada atenção à saúde de pessoas idosas [1]. Os problemas de saúde dos idosos desafiam os modelos tradicionais de cuidado e segundo Veras [2], envelhecer, independente da presença ou não de doenças crônicas, implica em alguma perda funcional como diminuição de vigor, força, prontidão, velocidade de reação sistêmica e eficiência metabólica.

No Brasil, os idosos representam $13 \%$ da população total, sendo a maior parte concentrada na região Sul (14,5\%) e a menor na região Norte (8,8\%). A faixa etária acima dos 60 anos concentra $11,8 \%$ da população total existente na região nordeste e $12,9 \%$ do estado da Paraíba [3]. A proporção do número de pessoas com 60 anos ou mais deve continuar se multiplicando, estipulando-se que em 2050, alcance dois bilhões de idosos, e a faixa acima de 80 anos ultrapassará a população de 400 milhões de pessoas [4].

Como consequência à maior expectativa de vida, tem-se observado o aumento exponencial das doenças crônicas não transmissíveis e, as sequelas e incapacidades oriundas dessas doenças demandam maior rigor no processo de cuidar diário, exigindo dos idosos e dos familiares maior atenção, tempo e dedicação. Quando se instalam dificuldades para 0 
autocuidado, ou havendo conflitos ou ausência dos familiares, associados dentre outros fatores à falta de condições para manutenção do cuidado, entram em cena as chamadas Instituições de Longa Permanência para Idosos (ILPI) como suporte social para essa população específica [5].

Nessas instituições, além de idosos com doenças crônicas em curso, geralmente se encontram idosos com capacidade funcional comprometida, especialmente idosos com alguma limitação física (pessoas com deambulação prejudicada que passam a maior parte ou o dia inteiro sentadas, e idosos acamados), verifica-se também que a precária mobilização associada a outros fatores de risco, torna-os vulneráveis ao desenvolvimento de lesões, como a lesão por pressão.

A lesão por pressão, nova nomenclatura, antes chamada de úlcera por pressão, é um dano localizado na pele e/ou tecido mole subjacente geralmente sobre proeminência óssea ou também pode estar relacionado a equipamentos médicos ou outro tipo de dispositivo. A lesão pode apresentar-se como pele intacta ou como úlcera aberta e pode ser dolorosa. Ocorre como um resultado de intensa e/ou prolongada pressão ou de pressão combinada com cisalhamento [6]. A tolerância do tecido mole à pressão e cisalhamento poderá ser afetada pelo microclima, nutrição, perfusão, doenças associadas e condição do tecido mole [7].

Estudos realizados entre idosos institucionalizados apontam uma prevalência de lesão por pressão que varia entre $18,8 \%$ e $26,6 \%$. Esse panorama evidencia a importância da prevenção dessas lesões pela equipe de saúde, em especial profissionais da enfermagem e os cuidadores formais de idosos das ILPI's, especificamente nos idosos com dependência funcional total ou parcial [8-10].

A princípio, torna-se importante explicar quem é o cuidador de idosos, uma pessoa membro ou não-membro familiar, com ou sem remuneração, alguém que cuida do idoso dependente ou doente, auxiliando nas atividades de vida diária como: alimentação, higiene pessoal, acompanhamento aos serviços de saúde ou outros que se fizerem necessários, acompanhamento da medicação oral de rotina, exceto realização de técnicas ou procedimentos identificados por profissões legalmente estabelecidas, a exemplo da Enfermagem [11].

No entanto, diferencia-se o tipo de cuidador de idosos conforme o sistema de cuidados: cuidador formal e cuidador informal. Cuidadores formais são pessoas que atuam de forma remunerada na assistência ao idoso, possuem vínculo empregatício, estão preparados e treinados para assistir ao idoso e familiares. E denomina-se cuidador informal a pessoa que atua de forma voluntária na assistência ao idoso, sem remuneração ou formação profissional específica, na maioria das vezes são membros diretos da família [12]. Nesse estudo, utilizaremos a expressão cuidador formal de idosos, para um melhor esclarecimento quanto ao tipo de cuidador em foco.

Portanto, o exercício da função de cuidador de idoso de forma remunerada é atributo do cuidador formal de idosos, e como função vinculada a trabalho. O Ministério do Trabalho e Emprego reconhece a função de cuidador como ocupação profissional, tendo por função primária conforme Classificação Brasileira de Ocupações (CBO) [13]: Cuidar de idosos, a partir de objetivos estabelecidos por instituições especializadas ou responsáveis diretos, zelando pelo bem-estar, saúde, alimentação, higiene pessoal, educação, cultura, recreação e lazer da pessoa assistida.

Igualmente, a Política Nacional de Saúde do Idoso [11] prevê algumas competências para os cuidadores de pessoas dependentes, e ressalta que a função do cuidador é acompanhar e auxiliar o idoso ajudando-o nas atividades de vida diária. No entanto, não se deve confundir com o profissional da enfermagem, por isso algumas técnicas e procedimentos não são autorizados de serem feitos pelo cuidador.

Projetos de Lei tramitam na Câmara dos Deputados e Senado Federal, e atualmente aguarda-se aprovação do Projeto de Lei (PL) 284/2011 [14] na Câmara Federal, já aprovado pelo Senado Federal, que apontam os requisitos para o exercício da profissão com 0 estabelecimento de escolaridade mínima e formação em cursos para cuidadores, discutem sobre as atribuições, e ampliam o âmbito de atuação, estendendo a profissão de cuidador ao contexto institucional.

Portanto, nas ILPI os cuidadores formais de idosos são agentes de cuidados de suma relevância, visto que conhecem mais o idoso sob seus cuidados considerando seu maior contato diário, além de que contribuem para o bem-estar e saúde destes, através do auxílio nas atividades funcionais, sendo considerados por alguns autores como integrantes da equipe de profissionais da saúde [15]. 
Assim, considerando que a ocorrência da lesão por pressão eleva a morbimortalidade do usuário, compromete a qualidade de vida da pessoa portadora da lesão e aumenta a sobrecarga de cuidados do cuidador, alguns cuidados preventivos básicos e específicos são necessários, bem como urgentes para a manutenção da integridade da pele do idoso e minimização da ocorrência dessas feridas. Levando em consideração essas premissas, questionou-se: Os cuidadores formais de idosos de ILPIs sabem o que é uma úlcera por pressão e as maneiras de preveni-la? Para responder ao questionamento supracitado, objetivase: avaliar o conhecimento dos cuidadores formais de idosos, de ILPIs, sobre UP e formas de prevenção dessa lesão.

\section{Material e métodos}

Trata-se de um estudo transversal de abordagem quanti-qualitativa, realizado com cuidadores formais de idosos em duas Instituições de Longa Permanência para Idosos (ILPIs), localizadas em João Pessoa, Paraíba.

As duas ILPIs somadas assistiam 103 idosos, em que 55 eram mulheres e 48 homens. O quadro de recursos humanos dessas instituições era bastante diversificado: uma enfermeira em cada ILPI, técnicos de enfermagem, médicos (maioria voluntários), nutricionistas, fisioterapeutas, assistentes sociais, psicólogas, auxiliar administrativo/secretária, gestores, auxiliares de serviços gerais e cuidadores de idosos. Ressalta-se que em uma das instituições aqueles que exerciam a função de cuidadores de idosos, também eram responsáveis pela limpeza das dependências da ILPI.

Neste estudo a população-alvo foram os cuidadores formais de idosos, que totalizavam dezesseis cuidadores de idosos nas duas instituições pesquisadas. Os cuidadores foram convidados para participar do estudo conforme os critérios de inclusão: atuar na ILPI na função de cuidador formal de idosos no mínimo há seis meses e não estar de férias e/ou licença médica durante a coleta de dados. A amostragem adotada foi a não probabilística, do tipo por conveniência, obteve-se uma amostra de quatorze cuidadores.

A coleta de dados foi realizada durante os meses de junho e julho de 2016, através da técnica de entrevista, para a qual foi elaborado pelas pesquisadoras um roteiro estruturado dividido em três partes, contemplando as seguintes variáveis: I. Perfil demográfico (sexo, idade, estado civil, escolaridade); II. Perfil profissional (curso de cuidador de idoso, curso técnico de enfermagem, tempo de atuação como cuidador de idosos, jornada de trabalho, número de idosos que cuida, atividades desenvolvidas na ILPI) e III. Conhecimento sobre prevenção de úlcera por pressão (entendimento sobre o que é úlcera por pressão, causas dessas lesões, fatores de risco para essas lesões, principais áreas corporais de ocorrência, formas/medidas de prevenção). Ressalta-se que o instrumento elaborado foi enviado para a apreciação de três docentes-pesquisadoras, uma da área da Enfermagem gerontológica e as demais eram membros do Grupo de Estudo e Pesquisa no Tratamento de Feridas (GEPEFE) da Universidade Federal da Paraíba, desta forma, após a avaliação algumas questões foram suprimidas e esclarecidas.

$\mathrm{Na}$ III Parte do instrumento que trata do conhecimento dos cuidadores de idosos sobre prevenção de úlcera por pressão, as autoras consideraram imperativo avaliar inicialmente o entendimento dos cuidadores sobre a lesão por pressão propriamente dita, desde o conceito do que são essas lesões, suas causas e área/localização anatômica, pois esses saberes concorrem para um melhor conhecimento da causa dessas lesões. Assim, seguindo essa ordem cognitiva, aproximou-se das reflexões dos cuidadores sobre os modos de prevenir ou evitar as lesões por pressão.

Para análise das variáveis relacionadas aos perfis demográficos e profissionais utilizouse a estatística descritiva, apresentados através de números absolutos e percentuais; de outro modo, os dados concernentes ao conhecimento dos cuidadores de idosos sobre lesão por pressão e sua prevenção foram analisados sob enfoque da abordagem quanti-qualitativa, para a qual foram consideradas três categorias: correta/completa, parcialmente correta e incorreta/incompleta. As definições para categorização das respostas, especificamente na formulação das questões referentes à III Parte do instrumento, foram construídas baseadas nas últimas diretrizes internacionais sobre prevenção de úlcera por pressão [7]. Tais detalhamentos são expostos no quadro 1.

Este estudo seguiu os procedimentos éticos e legais, sendo aprovado sob número 008950/2016 e CAAE 53192616.6.0000.5183 pelo Comitê de Ética e Pesquisa 5183- Hospital Universitário Lauro Wanderley/UFPB. Todos os participantes concordaram participar do estudo, 
assinando o Termo de Consentimento Livre e Esclarecido. Esta pesquisa foi realizada com base nos aspectos éticos em pesquisa envolvendo seres humanos, preconizados pela Resolução 466/12 do Conselho Nacional de Saúde [16].

Quadro 1 - Categorização das respostas dos cuidadores de idosos, conforme definições das últimas Diretrizes Internacionais sobre Úlcera por Pressão. João Pessoa/PB, 2016.

\begin{tabular}{|c|c|c|c|c|}
\hline & Conceito & Fatores de risco & $\begin{array}{l}\text { Principais áreas } \\
\text { corporais }\end{array}$ & Formas de prevenção \\
\hline Definições & $\begin{array}{l}\text { Uma lesão localizada na } \\
\text { pele e/ou tecidos } \\
\text { subjacentes, normalmente } \\
\text { sobre uma proeminência } \\
\text { óssea como resultado de } \\
\text { intensa e/ou prolongada } \\
\text { pressão ou de pressão } \\
\text { combinada com } \\
\text { cisalhamento. A tolerância } \\
\text { do tecido mole à pressão } \\
\text { e cisalhamento poderá ser } \\
\text { afetada pelo microclima } \\
\text { (umidade e temperatura), } \\
\text { nutrição, perfusão, } \\
\text { doenças associadas e } \\
\text { condição do tecido mole. }\end{array}$ & $\begin{array}{l}\text { Indivíduos } \\
\text { acamados e/ou } \\
\text { confinados a } \\
\text { cadeiras de rodas, } \\
\text { limitações de } \\
\text { mobilidade, } \\
\text { perfusão e } \\
\text { oxigenação } \\
\text { diminuídas, } \\
\text { umidade da pele, } \\
\text { aumento da } \\
\text { temperatura } \\
\text { corporal, idade } \\
\text { avançada, } \\
\text { percepção sensorial } \\
\text { diminuída. }\end{array}$ & $\begin{array}{l}\text { Áreas corporais de } \\
\text { maior risco para UP, } \\
\text { como a região } \\
\text { anatômica sacral, } \\
\text { calcâneo, ísquio, } \\
\text { trocânter, occipital, } \\
\text { escapular, maleolar e } \\
\text { regiões corporais } \\
\text { submetidas à pressão } \\
\text { por dispositivos como a } \\
\text { presença de cateteres, } \\
\text { tubos e drenos. }\end{array}$ & $\begin{array}{l}\text { Mudança de decúbito; } \\
\text { higienização da pele; manter } \\
\text { pele seca/ livre de umidade; } \\
\text { troca sistemática de fraldas; } \\
\text { hidratação da pele; uso de } \\
\text { materiais para alívio da } \\
\text { pressão; inspeção diária da } \\
\text { pele; suporte nutricional } \\
\text { adequado; manter lençóis } \\
\text { limpos e estirados; evitar } \\
\text { fricção/cisalhamento no } \\
\text { manejo com o paciente; } \\
\text { evitar massagens nas áreas } \\
\text { de proeminências ósseas; } \\
\text { capacitação profissional. }\end{array}$ \\
\hline $\begin{array}{l}\text { Correta / } \\
\text { Completa }\end{array}$ & $\begin{array}{l}\text { Respostas que } \\
\text { contemplem pelo menos } \\
\text { os seguintes pontos: } \\
\text { ferimento/lesão de pele; } \\
\text { Pressão/cisalhamento/ } \\
\text { fricção; isquemia. }\end{array}$ & $\begin{array}{l}\text { Respostas que } \\
\text { contemplem pelo } \\
\text { menos três fatores } \\
\text { de risco citados na } \\
\text { definição acima. }\end{array}$ & $\begin{array}{l}\text { Respostas que } \\
\text { contemplem pelo } \\
\text { menos três locais de } \\
\text { acometimentos citados } \\
\text { na definição acima. }\end{array}$ & $\begin{array}{l}\text { Respostas que contemplem } \\
\text { pelo menos cinco prevenções } \\
\text { citadas na definição acima. }\end{array}$ \\
\hline $\begin{array}{l}\text { Parcialmente } \\
\text { correta / } \\
\text { Incompletas }\end{array}$ & $\begin{array}{l}\text { Respostas que } \\
\text { contemplem pelo menos } \\
\text { um dos itens considerados } \\
\text { na categoria correta. }\end{array}$ & $\begin{array}{l}\text { Respostas que } \\
\text { contemplem pelo } \\
\text { menos um dos } \\
\text { fatores de risco da } \\
\text { categoria correta. }\end{array}$ & $\begin{array}{l}\text { Respostas que } \\
\text { contemplem pelo } \\
\text { menos um local de } \\
\text { ocorrência de UP, } \\
\text { dentre as áreas citadas } \\
\text { na categoria correta. }\end{array}$ & $\begin{array}{l}\text { Respostas que contemplem } \\
\text { menos de cinco ações } \\
\text { preventivas citadas na } \\
\text { categoria correta. }\end{array}$ \\
\hline Incorreta & $\begin{array}{l}\text { Respostas que não } \\
\text { contemplem nenhum dos } \\
\text { itens considerados na } \\
\text { categoria correta. }\end{array}$ & $\begin{array}{l}\text { Respostas que não } \\
\text { contemplem } \\
\text { nenhum dos itens } \\
\text { considerados na } \\
\text { categoria correta. }\end{array}$ & $\begin{array}{l}\text { Respostas que não } \\
\text { contemplem nenhum } \\
\text { dos itens considerados } \\
\text { na categoria correta. }\end{array}$ & $\begin{array}{l}\text { Respostas que não } \\
\text { contemplem nenhum dos } \\
\text { itens considerados na } \\
\text { categoria correta. }\end{array}$ \\
\hline
\end{tabular}

Os cuidadores de idosos entrevistados eram em sua maioria do sexo feminino (78,5\%), predominando pessoas solteiras (42\%), com faixa etária de 24 a 54 anos. Quanto à formação educacional e/ou profissional, $57 \%$ referiam ensino médio concluído, $50 \%$ informaram ter realizado curso de cuidador de idosos, e $28,5 \%$ fizeram o curso técnico de enfermagem.

No que se refere à ocupação desempenhada na ILPI, todos os pesquisados eram cuidadores formais de idosos, o tempo médio de atuação nessa atividade era de oito anos. Quanto à jornada de trabalho, os cuidadores referiram cumprir uma carga horária de oito horas por dia, distribuída em seis dias por semana e informaram ser responsáveis por cinco a treze idosos nas ILPIs. As atividades citadas pelos cuidadores como competências desenvolvidas nas instituições foram: banho nos idosos dependentes; asseio/higienização nos idosos acamados e incontinentes; auxílio aos idosos em suas necessidades diárias como vestir-se, alimentar-se, deambular e ir ao banheiro; passeio com os idosos; limpeza diária dos quartos e sanitários; troca de lençóis de cama; limpeza da instituição e servir refeições.

Quanto ao conhecimento dos cuidadores sobre a lesão por pressão e suas medidas de prevenção, conforme elucidado anteriormente na metodologia, as autoras do presente estudo acreditam que é de suma importância avaliar o entendimento sobre o que se trata uma lesão por pressão, pois compreender "o porquê" pactua com "o que fazer" como medida(s) preventiva(s) ou pelo menos colabora para o cuidador evitar algumas ações prejudiciais ou 
omissões que contribuam para o desenvolvimento da lesão. A seguir serão destacadas algumas falas dos entrevistados segundo os questionamentos realizados, as quais foram classificadas como respostas: Correta/completa, Parcialmente correta e Incorreta/incompleta.

O Quadro 2 apresenta algumas respostas sobre o que seria essa lesão, verificou-se que as respostas foram breves e sinalizaram tratar-se de ferimento/ ferida.

Quadro 2 - Respostas dos cuidadores formais relacionadas ao conceito de úlcera por pressão. João Pesso/PB, 2016.

\section{$O$ que é úlcera por pressão? *}

[E: 14] "É um ferimento causado pela pressão $\quad$ Correto (1/ 7,2\%)

do membro sob o colchão"

[E: 1] "É uma ferida de longo tempo, afinando $\quad$ Parcialmente correto (12/85,7\%)

transforma-se em escara"

[E: 2] "É um ferimento que acontece

principalmente em idoso."

[E: 13] "Quando a pele começa a ficar bem

fininha"

Fonte: Pesquisa Própria. João Pessoa, 2016; * Durante a entrevista, para facilitar a comunicação sobre o termo "úlcera por pressão", a pergunta foi elaborada da seguinte forma: "Você sabe o que é úlcera por pressão ou escara como eram chamadas no passado?".

Concernente aos fatores de risco para o desenvolvimento da lesão por pressão, a maioria dos cuidadores responderam de forma parcialmente correta, posto que apenas dois fatores foram mais citados: a imobilidade e higienização. Outros fatores de risco foram mencionados de maneira isolada (Quadro 3). Ressalta-se que nenhum cuidador referiu desconhecer qualquer fator de risco, ou mesmo não respondeu aos questionamentos, de forma, que nenhuma resposta foi considerada incorreta.

Quadro 3 - Respostas dos cuidadores formais relacionadas ao questionamento: "O que leva 0 idoso a desenvolver a úlcera por pressão?". João Pessoa/PB, 2016.

[E: 12] "Muito tempo em uma posição só, $\quad$ Correta $(3 / 21,4 \%)$

permanecer urinado e calor"

[E: 14] "Ficar deitado em uma só posição.

Ficar deitado sobre o lençol dobrado. Uso de

fralda. Excesso de urina".

[[E:7] "Ficar sujo de resíduos. Ficar parado na cama"

[E:9] "Ficar muito tempo em uma posição só.

Higienização mal feita".

Fonte: Pesquisa Própria. João Pessoa, 2016.

Referente às áreas/locais no corpo de ocorrência da lesão por pressão, poucas áreas foram citadas pelos pesquisados e, portanto, a maior parte das respostas foram consideradas parcialmente corretas, Quadro 4.

Quadro 4 - Respostas dos cuidadores formais relacionadas ao questionamento: "Quais os locais de ocorrência de úlcera por pressão nos idosos?". João Pessoa/PB, 2016.

E: 14] "Calcâneo. Sacral. Nuca. Cotovelo. $\quad$ Correta (4/ 28,6\%)

Joelho".

[E: 11] "Região Sacral. Costas. Calcâneo".

[E:13] "Na região sacral".

[E 6] "Mais no glúteo, na parte anterior do joelho".

Fonte: Pesquisa Própria. João Pessoa, 2016.

Quanto às formas de prevenção das lesões, os cuidadores apontaram média de duas a três ações como medidas preventivas, assim sendo, todas as respostas (100\%) foram classificadas em parcialmente corretas/incompletas (Quadro 5). 
Quadro 5 - Respostas dos cuidadores formais relacionadas ao questionamento: "Você conhece alguma forma para evitar (prevenir) essas feridas?" João Pessoa/PB, 2016.

\begin{tabular}{|l|l|}
\hline [E:9] "Hidratar muito com água. Mudança de & Incompleto (14/ 100\%) \\
posição. Sempre dar banho" \\
[E:3] "Sempre dar banho. Sempre trocar fralda. \\
Mudar de posição."
\end{tabular}

Fonte: Pesquisa Própria. João Pessoa, 2016.

Neste estudo, a maioria dos cuidadores formais de idosos era mulheres, dentro da faixa etária de idade ativa, ensino médio completo, semelhantes aos perfis encontrados em outras pesquisas com cuidadores formais de idosos $[17,18]$. Verificou-se que a forte presença feminina entre os cuidadores segue a tradição histórica e cultural da mulher como cuidadora nata.

Identificou-se que metade dos pesquisados realizaram curso específico para cuidador de idosos, assim, a realidade encontrada compactua com o perfil de cuidadores formais de outros estudos $[18,19]$. Esse achado ainda se repete em nosso meio, devido a não exigência de curso de formação de cuidadores de idosos no ato da contratação do trabalhador, visto que a legislação brasileira ainda não aprovou a alteração da função de cuidador de ocupação para profissão regulamentada. Quanto aos dispositivos legais que respaldem os cuidadores de idosos enquanto profissão, tramitam-se projetos de lei discutindo: requisitos, atribuições e questões trabalhistas.

Ainda sobre a formação profissional dos cuidadores, o PL 4702/2012 [20], proposto pelo Senado Federal, acrescenta aos PL 2178/2011 [21] e PL 284/2011 [14], e descreve de forma mais completa as proposições quanto à qualificação do profissional cuidador. Estabelece que está habilitado para o exercício desta profissão aquele que tiver concluído o curso de formação de cuidador de pessoa idosa. São dispensadas da exigência de conclusão de curso de formação as pessoas que estejam atuando na função há no mínimo dois anos, quando a Lei entrar em vigor. Contudo, prevê que nos cinco anos seguintes o cuidador deverá realizar a referida formação ou concluir o programa de certificação de saberes reconhecido pelo Ministério da Educação.

Mas, considerando a atual prescrição legal derivada da CBO [13], dentre as atividades passíveis de serem desenvolvidas pelos cuidadores, estão: promover o bem-estar do idoso, cuidar de sua saúde, alimentação, higiene pessoal e de seu ambiente domiciliar e institucional. Como também: estimulá-lo e auxiliá-lo no desempenho de atividades de vida diária; acompanhá-lo em atividades externas; incentivá-lo à participação nas atividades de cultura e educação.

Retornando aos achados da atual pesquisa, com relação ao conhecimento dos cuidadores de idosos sobre a úlcera por pressão/ lesão por pressão (conceito, áreas de ocorrência e fatores de risco para a lesão) e medidas preventivas para a ocorrência da ferida, verificou-se que grande parte das respostas foi classificada como parcialmente corretas, demostrando fragilidades no conhecimento. Desta forma, enfatiza-se a inter-relação existente entre conhecer o que é a lesão, como ela pode surgir e em que locais, de modo que esse entendimento favoreça o emprego de ações preventivas adequadas. Autores apontam que o déficit de conhecimento de cuidadores relacionada às suas ações, dificulta a compreensão do que acontece com o próprio idoso [22].

Destaca-se que a manutenção de cuidados de higiene e mobilização do idoso são ações que podem ser efetivadas pelo cuidador e, portanto, realizar ações seguras e eficazes que ajudem na manutenção da integridade da pele do idoso concorre para prevenir lesões. No Guia Prático do Cuidador [23] elaborado pelo Ministério da Saúde, dois capítulos mencionam a lesão por pressão (na época denominou-se de úlcera de pressão/ escara): o Capítulo 10 Cuidados no domicílio para pessoas acamadas ou com limitações físicas e, de modo específico para a prevenção - o Capítulo 23 - Úlcera de Pressão/ Escaras/ Feridas.

Vale ressaltar que se observa um déficit de saberes sobre essas lesões, não somente por parte dos cuidadores, mas também de profissionais da enfermagem, conforme identificados em outros estudos $[24,25]$. Por outro lado, torna-se importante ressaltar que o aparecimento desse tipo de lesão é visto como indicador negativo da qualidade assistencial, conforme declarado na Política Nacional de Segurança do Paciente [26]. 
O desenvolvimento de UP em idosos é algo evitável, e para minimizar a ocorrência dessas lesões, torna-se necessário conhecer os fatores de risco; por conseguinte, conhecendo quais são esses fatores, os profissionais responsáveis pelo cuidado devem colocar em prática medidas preventivas em prol do bem-estar dessa clientela [25].

Diante do exposto, suscita à reflexão que o conhecimento desse ser cuidador é fundamental para a manutenção da integridade da pele dos idosos sob seus cuidados, de modo contrário, a qualidade do cuidado relacionado à prevenção de UP pode estar prejudicado, se o conhecimento e a habilidade deles não estiverem adequadamente conduzidos. Logo, o conhecimento técnico-científico tem como base identificar qualquer ocorrência na questão da integridade da pele, através de um olhar individualizado ao idoso [9].

Um estudo com cuidadores identificou necessidade de mais conhecimentos para cuidar dos idosos, e em concordância com as autoras, quando afirmaram que a dificuldade existente sobre conhecimentos específicos poderia ser solucionada ou minimizada através de capacitações voltadas aos cuidadores, de modo a se alcançar cuidados conscientes, efetivos e integrais no cotidiano da assistência aos idosos [27].

\section{Conclusão}

No presente estudo, pode-se perceber que há um déficit de conhecimento relacionado à lesão por pressão entre os cuidadores formais de idosos que atuam nas ILPI pesquisadas. Embora tenha sido realizado em apenas duas ILPIs, os resultados ora apresentados não possuem critérios de generalizações, podendo esta ser uma limitação do presente estudo, contudo o fato de corroborar alguns estudos realizados aponta que a situação local é condizente com outras realidades de nosso país.

Ainda que novos estudos sejam necessários para sanar as possíveis lacunas aqui evidenciadas, verificou-se como urgente a viabilização de intervenções educativas fundamentadas no cuidado à saúde do idoso, especificamente na capacitação dos cuidadores para prevenção das lesões por pressão.

\section{Referências}

1. Silva A, Dal Prá KR. Envelhecimento populacional no Brasil: o lugar das famílias na proteção aos idosos. Rev Argumentum Vitória 2014;16(1):99-115.

2. Veras R. A urgente e imperiosa modificação no cuidado à saúde da pessoa idosa. Rev Bras Geriatr Gerontol 2015;18(1):5-6.

3. Brasil. Ministério do Planejamento, orçamento e gestão; Instituto Brasileiro de Geografia e Estatística; diretoria de pesquisas; coordenação de população e indicadores sociais. Sínteses de indicadores sociais: uma análise das condições de vida da população brasileira. Estudos \& pesquisas, informações demográficas e socioeconômicas; $n$. 32. Rio de Janeiro: IBGE; 2013.

4. Organização das Nações Unidas (ONU). A ONU e as pessoas idosas 2016. [citado 2017 Abr 26]. Disponível em: https://nacoesunidas.org/acao/pessoas-idosas/.

5. Cavalcanti $A D$. Envelhecimento e institucionalização: uma revisão bibliográfica à luz da promoção da saúde. Revista da Faculdade de Ciências Humanas e Saúde 2013;16(4):159-74.

6. National Pressure Ulcer Advisory Panel, European Pressure Ulcer Advisory Panel e Pan Pacific Pressure Injury Alliance. Announces a change in terminology from pressure ulcer to pressure injury and updates the stages of pressure injury. 2016. [citado 2017 Abr 28]. Disponível em: http://www.npuap.org

7. National Pressure Ulcer Advisory Panel, European Pressure Ulcer Advisory Panel e Pan Pacific Pressure Injury Alliance. Prevention and Treatment of Pressure Ulcers: Quick Reference Guide. 2014. [citado 2017 Abr 28]. Disponível em: http://www.npuap.org/

8. Vilanova GC, Takebayashi RB, Yoshitome AY, Blanes L. Avaliação de risco e prevalência da úlcera por pressão em idosos residentes em uma Instituição de longa permanência filantrópica da cidade de São Paulo. Rev Estima 2009;7(1):1-9.

9. Freitas MC, Medeiros ABF, Guedes MVC, Almeida PC, Galiza FT, Nogueira JM. Úlcera por pressão em idosos institucionalizados: análise da prevalência e fatores de risco. Rev Gaúcha Enferm 2011;32(1):143-50. 
10. Andrade EMLR, Sousa JERB, Rabelo CBM, Silva HF, Rabelo CBM, Bezerra SMG et al. Fatores de risco e ocorrência de úlcera por pressão em idosos institucionalizados. Rev Enferm UFPI 2012;1(1):36-41.

11. Brasil. Ministério da Saúde. Política Nacional de Saúde da Pessoa Idosa. Portaria $n^{\circ}$ 2.528, de outubro de 2006. Brasília: Ministério da Saúde; 2006.

12. Born T. Cuidar melhor e evitar a violência - manual do cuidador da pessoa idosa. Brasília: Secretaria Especial dos Direitos Humanos, Subsecretaria de Promoção e Defesa dos Direitos Humanos; 2008.

13. Brasil. Portaria ํo 397, de 10 de dezembro de 2002. Institui a Classificação Brasileira de Ocupações. Brasília: Ministério do Trabalho e Emprego; 2002.

14. Brasil. Senado Federal. Projeto de Lei oㅡ 284, de 2011. Brasília, DF.

15. Sampaio AMO, Rodrigues FN, Pereira VG, Rodrigues SM, Dias CA. Cuidadores de idosos: percepção sobre o envelhecimento e sua influência sobre o ato de cuidar. Estudos e Pesquisas em Psicologia 2011;11(20):590-613.

16. Brasil. Ministério da Saúde. Conselho Nacional de Saúde. Resolução no 466, de 12 de dezembro de 2012. Brasília: Ministério da Saúde; 2012.

17. Silva I, Machado F, Ferreira M, Rodrigues M. Formação profissional de cuidador de idosos atuantes em instituições de longa permanência. Rev Holos 2015;8:342-56.

18. Lopes RA, Mitre NCD, Coelho MGM, Queiroz BZ. Perfil dos cuidadores das instituições de longa permanência para idosos de Itaúna - MG. Rev ConsScientiae Saúde 2012;11(2):338-44.

19. Lampert CDT, Scortegagna SA, Grzybovski D. Dispositivos legais no trabalho de cuidadores: aplicação em instituições de longa permanência. Rev REAd 2016;22(3):360-80.

20. Brasil. Projeto de Lei $n^{\circ} 4702$, de 2012. Dispõe sobre o exercício da profissão de cuidador de pessoa idosa e dá outras providências. Senado Federal. Brasília, DF. 2012.

21. Brasil. Projeto de Lei $n-2178$, de 2011. Dispõe sobre o exercício da profissão de cuidador. Câmara dos Deputados. Brasília, DF. 2011.

22. Araújo JS, Vidal GM, Brito FN, Gonçalves DCA, Leite DKM, Dutra CDT, et al. Perfil dos cuidadores e as dificuldades apresentadas no cuidado ao idoso, em Ananindeua, PA. Rev Bras Geriatr 2013;16(1):149-58.

23. Brasil. Ministério da Saúde, Secretaria de Atenção à Saúde, Secretaria de Gestão do Trabalho e da Educação na Saúde. Guia prático do cuidador. Brasília: Ministério da Saúde; 2008.

24. Miyazaki MY, Caliri MHL, Santos C.B. Conhecimento dos profissionais de enfermagem sobre prevenção da úlcera por pressão. Rev Latinoam Enferm 2010;18(6):1-10.

25. Ferreira DPC, Guarda FR. A percepção dos cuidadores familiares acerca da úlcera por pressão em idosos. Rev Enferm UFPE on line 2015;9(11):321-7.

26. Brasil. Ministério da Saúde. Documento de referência para o Programa Nacional de Segurança do Paciente / Ministério da Saúde; Fundação Oswaldo Cruz; Agência Nacional de Vigilância Sanitária. Brasília: Ministério da Saúde; 2014.

27. Colomé ICS, Marqui ABT, Jahn AC, Resta DG, Carli R, Winck MT, et al. Cuidar de idosos institucionalizados: características e dificuldades dos cuidadores. Rev Eletr Enferm 2011;13(2):306-12. 